\title{
Atomic-scale simulations of the mechanical deformation of nanocrystalline metals
}

\author{
Schiøtz, Jakob; Vegge, Tejs; Di Tolla, Francesco; Jacobsen, Karsten Wedel
}

Published in:

Physical Review B

Link to article, DOI:

10.1103/PhysRevB.60.11971

Publication date:

1999

Document Version

Publisher's PDF, also known as Version of record

Link back to DTU Orbit

Citation (APA):

Schiøtz, J., Vegge, T., Di Tolla, F., \& Jacobsen, K. W. (1999). Atomic-scale simulations of the mechanical deformation of nanocrystalline metals. Physical Review B, 60(17), 11971-11983.

https://doi.org/10.1103/PhysRevB.60.11971

\section{General rights}

Copyright and moral rights for the publications made accessible in the public portal are retained by the authors and/or other copyright owners and it is a condition of accessing publications that users recognise and abide by the legal requirements associated with these rights.

- Users may download and print one copy of any publication from the public portal for the purpose of private study or research.

- You may not further distribute the material or use it for any profit-making activity or commercial gain

- You may freely distribute the URL identifying the publication in the public portal

If you believe that this document breaches copyright please contact us providing details, and we will remove access to the work immediately and investigate your claim. 


\title{
Atomic-scale simulations of the mechanical deformation of nanocrystalline metals
}

\author{
J. Schiøtz, ${ }^{*}$ T. Vegge, ${ }^{*}$ F. D. Di Tolla, ${ }^{\dagger}$ and K. W. Jacobsen \\ Center for Atomic-scale Materials Physics (CAMP) and Department of Physics, Technical University of Denmark, \\ DK-2800 Lyngby, Denmark
}

(Received 11 February 1999; revised manuscript received 11 June 1999)

\begin{abstract}
Nanocrystalline metals, i.e., metals in which the grain size is in the nanometer range, have a range of technologically interesting properties including increased hardness and yield strength. We present atomic-scale simulations of the plastic behavior of nanocrystalline copper. The simulations show that the main deformation mode is sliding in the grain boundaries through a large number of uncorrelated events, where a few atoms (or a few tens of atoms) slide with respect to each other. Little dislocation activity is seen in the grain interiors. The localization of the deformation to the grain boundaries leads to a hardening as the grain size is increased (reverse Hall-Petch effect), implying a maximum in hardness for a grain size above the ones studied here. We investigate the effects of varying temperature, strain rate, and porosity, and discuss the relation to recent experiments. At increasing temperatures the material becomes softer in both the plastic and elastic regime. Porosity in the samples result in a softening of the material; this may be a significant effect in many experiments. [S0163-1829(99)05941-X]
\end{abstract}

\section{INTRODUCTION}

The modeling of the mechanical properties of everyday materials is a very challenging problem. The main difficulty is the vastly different length and time scales at which the various processes occur during deformation-ranging from the Angström and subpicosecond scales of the atomic processes, to beyond the millimeter and second scales of the macroscopic deformation. Naturally, very different modeling techniques are required to model phenomena at so different scales. Atomic-scale simulations (typically molecular dynamics) can handle time scales of up to a few nanoseconds and system sizes of up to $10^{8}$ atoms, ${ }^{1,2}$ although one is typically limited to significantly smaller system sizes and simulation times by the available computer resources, and by the need to repeat the simulations at different conditions.

In spite of these limitations atomic-scale simulations are gaining increasing importance in materials science by being applied to selected subproblems or to problems where the natural length scale is in the nanometer range. ${ }^{1-5}$

One such atomic-scale problem is the mechanical deformation of nanocrystalline metals, i.e., metals where the grain size is in the nanometer range. Nanocrystalline metals have recently received much interest because they may have mechanical, chemical and physical properties different from their coarse-grained counterparts. For example, the hardness and yield stress may increase five to ten times when the grain size is reduced from the macroscopic to the nanometer range. ${ }^{6-9}$

Recently, computer simulations of the structure ${ }^{10-14}$ of nanocrystalline metals and semiconductors, and of their elastic $^{11}$ and plastic ${ }^{15-24}$ properties, have appeared in the literature. In previous papers, we described the plastic deformation of nanocrystalline copper at zero temperature. ${ }^{21,22}$ In this paper we focus on the elastic and plastic properties of nanocrystalline metals, in particular copper, at finite temperature. We find that the materials have a very high yield stress, and that the yield stress decrease with decreasing grain size (reverse Hall-Petch effect). The main deformation mode is found to be localized sliding in the grain boundaries.

The high yield stress and hardness of nanocrystalline metals is generally attributed to the Hall-Petch effect, ${ }^{25,26}$ where the hardness increases with the inverse square root of the grain size. The Hall-Petch effect is generally assumed to be caused by the grain boundaries acting as barriers to the dislocation motion, thus hardening the material. The detailed mechanism behind this behavior is still under debate. ${ }^{27-30} \mathrm{~A}$ cessation or reversal of the Hall-Petch effect will therefore limit the maximal hardness and strength that can be obtained in nanocrystalline metals by further refining of the grain size. There are a number of observations of a reverse Hall-Petch effect, i.e., of a softening when the grain size is reduced. ${ }^{31-34}$ The interpretation of these results have generated some controversy. It is at present not clear if the experimentally reported reverse Hall-Petch effect is an intrinsic effect or if it is caused by reduced sample quality at the finest grain sizes. The computer simulations presented here show that an intrinsic effect is clearly possible.

The structure of the paper is as follows. In Sec. II we discuss the setup of the nanocrystalline model systems. Section III discusses the simulation and analysis methods used. The simulation results are presented in Sec. IV, and subsequently discussed in Sec. V.

\section{SIMULATION SETUP}

In order to obtain realistic results in our simulations, and to be able to compare our simulations with the available experimental data, we have attempted to produce systems with realistic grain structures. Unfortunately, the microscopic structure is not very well characterized experimentally, and depends on the way the nanocrystalline metal was prepared. We have tried to create systems that mimic what is known about the grain structure of nanocrystalline metals generated by inert gas condensation. The grains seem to be almost equiaxed, separated by narrow grain boundaries. The 
grains are essentially dislocation free. The grain-size distribution is $\log$ normal. $6,35,36$

\section{A. Construction of the initial configuration}

In our simulations the grains are produced using a Voronoi construction: ${ }^{37}$ a set of grain centers are chosen at random, and the part of space closer to a given center than to any other center is filled with atoms in a randomly rotated face-centered-cubic (fcc) lattice. Periodic boundary conditions are imposed on the computational cell. This procedure generates systems without texture and with random grain boundaries. Effects of texture could easily be included by introducing preferred orientations of the grains. ${ }^{19}$ In the limit of a large number of grains, the Voronoi construction will generate a grain-size distribution close to a log-normal distribution. ${ }^{38}$

In the grain boundaries thus generated, it is possible that two atoms from two different grains get too close to each other. In such cases one of the atoms is removed to prevent unphysically large energies and forces as the simulation is started. To obtain more relaxed grain boundaries the system is annealed for 10000 timesteps $(50 \mathrm{ps})$ at $300 \mathrm{~K}$, followed by an energy minimization. This procedure is important to allow unfavorable local atomic configurations to relax.

To investigate whether the parameters of the annealing procedure are critical, we have annealed the same system for 50 and $100 \mathrm{ps}$ at $300 \mathrm{~K}$, and for $50 \mathrm{ps}$ at $600 \mathrm{~K}$. We have compared the mechanical properties of these systems with those of an identical system without annealing. We find that the annealing is important (the unannealed system was softer), but the parameters of the annealing are not important within the parameter space investigated.

A similar generation procedure has been reported by Chen, ${ }^{10}$ by D'Agostino and Van Swygenhoven, ${ }^{15}$ and by Van Swygenhoven and Caro. ${ }^{16,17}$ A different approach was proposed by Phillpot, Wolf, and Gleiter: ${ }^{11,12}$ a nanocrystalline metal is generated by a computer simulation where a liquid is solidified in the presence of crystal nuclei, i.e., small spheres of atoms held fixed in crystalline positions. The system was then quenched, and the liquid crystallized around the seeds, thus creating a nanocrystalline metal. In the reported simulations, the positions and orientations of the seeds were deterministically chosen to produce eight grains of equal size and with known grain boundaries, but the method can naturally be modified to allow randomly placed and oriented seeds. The main drawback of this procedure is the large number of defects (mainly stacking faults) introduced in the grains by the rapid solidification. The stacking faults are clearly seen in the resulting nanocrystalline metal (Fig. 7 of Ref. 11). The appearance of a large number of stacking faults was also seen in the solidification of large clusters even if the cooling is done as slowly as possible in atomistic simulations. ${ }^{39,40}$

\section{B. Structures}

A typical system (after annealing and cooling to zero Kelvin) with a grain size of $5.2 \mathrm{~nm}$ is shown in Fig. 1(a). The atoms have been color coded according to the local crystal structure, as determined by the common neighbor analysis (see Sec. III C). In Fig. 2 the radial distribution function a:

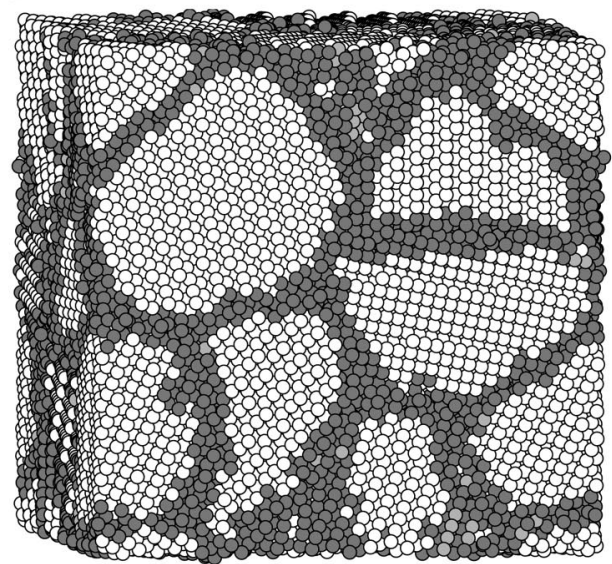

b:

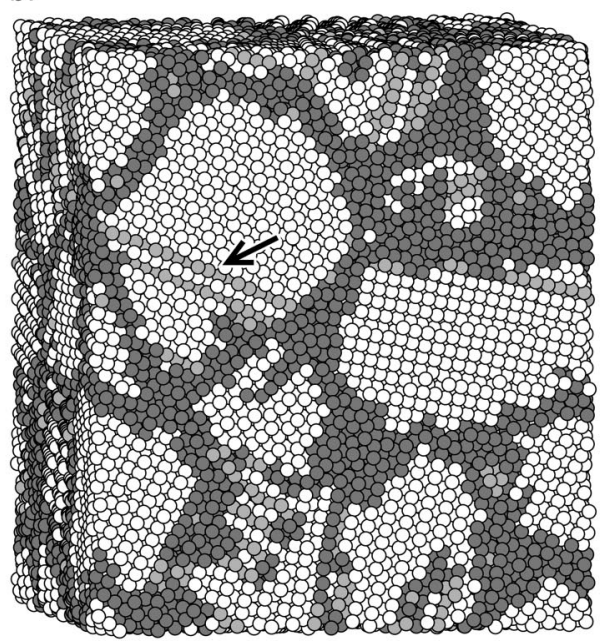

FIG. 1. The initial (a) and final (b) configuration of a nanocrystalline copper system deformed $10 \%$ at $300 \mathrm{~K}$. The system contains approximately 100000 atoms arranged in 16 grains, giving an average grain diameter of $5.2 \mathrm{~nm}$. Atoms are color-coded according to the local crystalline order. White atoms are in a perfect fcc environment. Light grey atoms are in local hep order, which for example corresponds to stacking faults. Atoms in any other environment (in grain boundaries and dislocation cores) are colored dark grey. The arrow marks an extrinsic stacking fault that appeared during the deformation. It was created by two Shockley partial moving through the grain on adjacent crystal planes.

(RDBF) $g(r)$ for the same system is shown. It is defined as the average number of atoms per volume at the distance $r$ from a given atom. The RDBF is seen to differ from that of a perfect fcc crystal in two ways. First, the peaks are not sharp delta functions, but are broadened somewhat. This broadening is in part due to strain fields in the grains (probably originating from the grain boundaries), and in part due to atoms in or near the grain boundaries sitting close to (but not at) the lattice positions. The second difference is seen in the inset: the RDBF does not go to zero between the peaks. It is the signature of some disorder which in this case comes from the grain boundaries.

Experimentally, information about the RDBF can be obtained from x-ray-absorption fine-structure (XAFS) measurements. This method has been used to measure the average coordination number of $\mathrm{Cu}$ atoms in nanocrystalline $\mathrm{Cu}$, finding coordination numbers of $11.8 \pm 0.3$ and $11.9 \pm 0.3$ in 


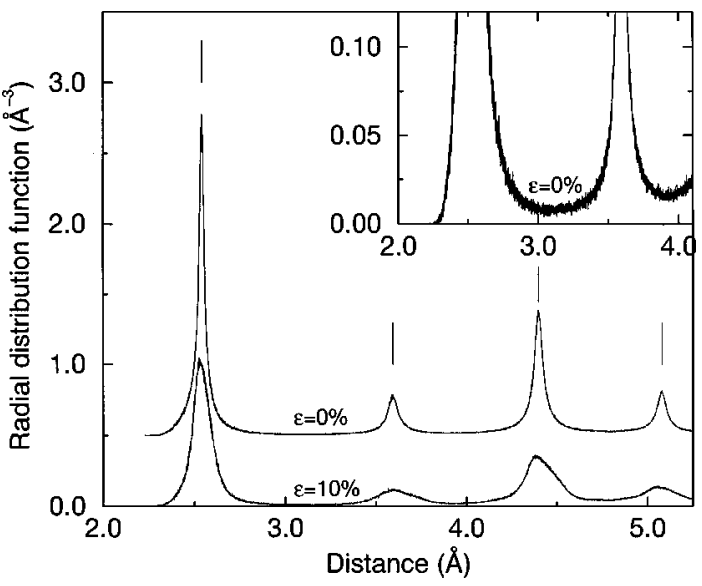

FIG. 2. The radial distribution function $g(r)$ for the system in Fig. 1(a) at $T=0$, before and after $10 \%$ deformation. The curve for the undeformed sample $(\varepsilon=0 \%)$ has been displaced 0.5 units upwards. $g(r)$ is the average number of atoms per volume at the distance $r$ from a given atom. The marks above the peaks indicate the positions of the (infinitely sharp) peaks in a perfect crystal at $T=0$. The inset shows that $g(r)$ does not vanish between the first and second peak as it does for a perfect crystal. This contribution comes from the grain boundaries.

samples with 34- and 13-nm grain size, respectively. ${ }^{41}$ From these results the average coordination number of the atoms in the grain boundaries was estimated to $11.4 \pm 1.2$, i.e., within the experimental uncertainty it is the same as in the bulk. Integrating the first peak of the calculated RDBF (Fig. 2, $\varepsilon$ $=0 \%$ ) gives an average coordination number of 11.9 \pm 0.15 . As the RDBF does not go to zero between the first two peaks, it is not clear where the upper limit of the integration should be chosen, hence the uncertainty. The value given is for an upper limit of $3.125 \AA$. There is thus excellent (but perhaps rather trivial) agreement between the calculated and the experimental coordination numbers.

Numerical studies have shown that the Voronoi construction results in a grain-size distribution that it is well described by a log-normal distribution (although for more than 5000 grains a two-parameter gamma distribution gives a better fit). ${ }^{38}$ In Fig. 3 we show the grain size distributions in our simulations with intended average grain sizes of 3.28 and $5.21 \mathrm{~nm}$. The observed distributions are consistent with lognormal distributions, although due to the rather low number of grains it is not possible to distinguish between a lognormal or a normal distribution.

\section{SIMULATION METHODS}

We model the interactions between the atoms using a many-body effective medium theory (EMT) potential. ${ }^{42,43}$ EMT gives a realistic description of the metallic bonding, in particular in fcc metals and their alloys. Computationally, it is not much more demanding than pair potentials, but due to its many-body nature it gives a far more realistic description of the materials properties.

The systems can be deformed by rescaling the coordinates along a direction in space (in the following referred to as the $z$ direction). During this deformation either a conventional molecular-dynamics (MD) algorithm or a minimization algo-

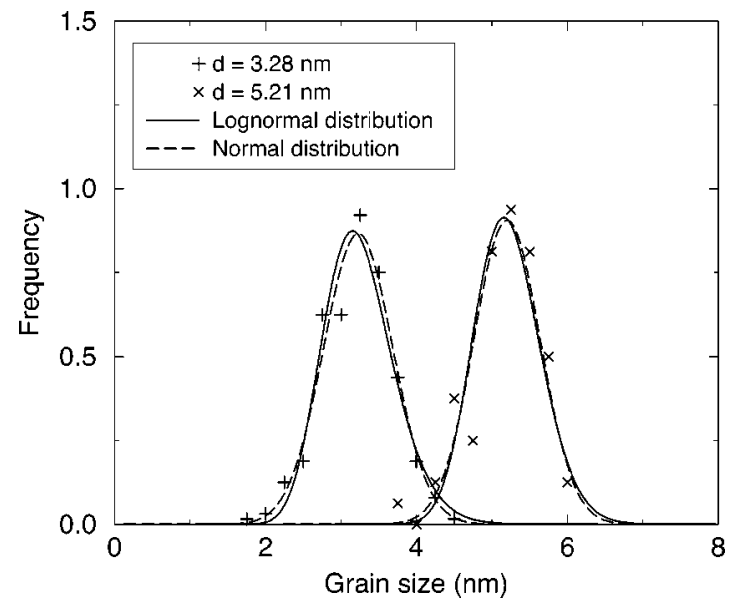

FIG. 3. The grain-size distributions obtained from the Voronoi construction. Grain-size distributions are shown for simulations with intended average grain sizes of 3.28 and $5.21 \mathrm{~nm}$. In both cases the input configurations from four simulations were used to calculate the distributions. Fits to log-normal (solid) and normal (dashed) distributions are shown. Both distributions fit the data well.

rithm is used to update the atomic positions in response to the deformation.

\section{A. Molecular dynamics at finite temperature}

At finite temperatures a conventional molecular-dynamics algorithm is used, where the Newtonian equations of motion for the atoms are solved numerically. Before the deformation is applied the system is heated to the desired temperature by a short molecular-dynamics simulations using Langevin dynamics, ${ }^{44}$ i.e., where a friction and a fluctuating force is added to the equation of motion of the atoms. When the desired temperature has been reached (after approximately $10 \mathrm{ps}$ ), the simulations is performed using the velocity Verlet algorithm. ${ }^{45}$ We use a timestep of $5 \mathrm{fs}$, safely below the value where the dynamics becomes unstable. During the deformation process the internal energy increases by the work performed on the system. This amounts in practice to a small heating of the system of the order of $\sim 30 \mathrm{~K}$.

At each timestep the system is deformed by a tiny scaling of the coordinates, the $z$ coordinates are multiplied by $1+\epsilon$, the $x$ and $y$ coordinates by $1-\nu \epsilon$, where $\epsilon$ is a very small number, chosen to produce the desired deformation rate. ${ }^{46}$ The constant $\nu$ is an "approximate Poisson's ratio." A Monte Carlo algorithm is used to optimize the two lateral dimensions of the system: after every 20th time step a change in the lateral dimensions is proposed and the resulting change in total energy is calculated. The proposed change is accepted based on the usual Metropolis criterion. ${ }^{44}$ In this way the exact value chosen for $\nu$ becomes uncritical, as the Monte Carlo algorithm governs the contraction in the lateral directions. The use of $\nu \neq 0$ is just for computational efficiency. We used $\nu=0.4$ as this was between the optimal value in the part of the simulation where elastic deformation dominates (Poisson's ratio $\approx 0.3$ ) and in the part where plastic deformation dominates $(\nu \approx 0.5$ as volume is then conserved). 


\section{B. Minimization procedure}

To simulate deformation at zero temperature a minimization procedure was used to keep the system in or near a local minimum in energy at all times. The deformation and minimization were done simultaneously. The minimization algorithm is a modified molecular dynamics simulation. ${ }^{47}$ After each MD time step the dot product between the momentum and the force is calculated for each atom. Any atom where the dot product is negative gets its momentum zeroed, as it is moving in a direction where the potential energy is increasing. This minimization procedure quickly brings the system close to a local minimum in energy, but a full convergence is not obtained, as it would require a number of time steps at least as great as the number of degrees of freedom in the system. However, we find only little change in the development of the system, when we increase the number of minimization steps.

A few simulations were repeated using a conjugate gradient (CG) minimization instead of the MD minimization algorithm. The two algorithms were approximately equally efficient in these simulations, provided that the CG algorithm was restarted approximately every 20 line minimizations. Otherwise the CG algorithm will not minimize twice along the same direction in the $3 N$-dimensional configuration space.

\section{Analysis of results}

While the simulation is performed, the stress field is regularly computed. The stress tensor is the derivative of the free energy of the system with respect to the strain. The effective medium theory allows us to define an energy per atom, which allows us to define an "atomic" stress for each atom. The stress is a suitable derivative of the energy with respect to the interatomic distances: ${ }^{48-50}$

$$
\sigma_{i, \alpha \beta}=\frac{1}{\mathrm{v}_{i}}\left(-\frac{p_{i, \alpha} p_{i, \beta}}{m_{i}}+\frac{1}{2} \sum_{j \neq i} \frac{\partial E_{\mathrm{pot}}}{\partial r_{i j}} \frac{r_{i j, \alpha} r_{i j, \beta}}{r_{i j}}\right),
$$

where $\sigma_{i, \alpha \beta}$ is the $\alpha, \beta$ component of the stress tensor for atom $i, \mathrm{v}_{i}$ is the volume assigned to atom $i\left(\sum_{i} \mathrm{v}_{i}=V\right.$, where $V$ is the total volume of the system), $m_{i}$ is the mass of atom $i, p_{i, \alpha}$ is the $\alpha$ component of its momentum, and $r_{i j}$ is the distance between atoms $i$ and $j$ ( $r_{i j, \alpha}$ is a component of the vector from atom $i$ to $j$ ).

The atomic stress tensor cannot be uniquely defined. Equation 1 is based on the Virial theorem, but other definitions are possible. ${ }^{51-53}$ When the atomic stress is averaged over a region of space the various definitions quickly converge to a macroscopic stress field. ${ }^{52,53}$ During the simulation, stress-strain curves are calculated by averaging the atomic stresses over the entire system.

To facilitate the analysis of the simulations the local atomic order was examined using an algorithm known as common neighbor analysis (CNA). ${ }^{54,55}$ In this algorithm the bonds between an atom and its nearest neighbors are examined to determine the crystal structure. We have used CNA to classify atoms into three classes: fcc, hcp, and "other,' i.e., atoms which are neither in a local fcc nor hep environment.

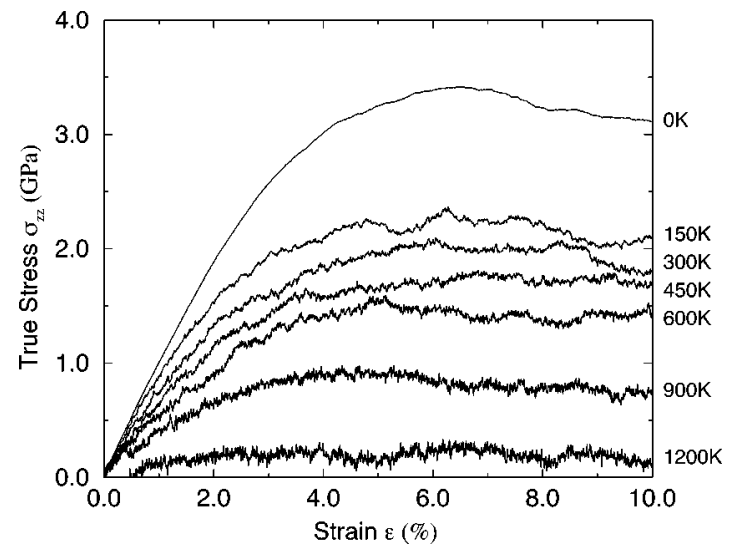

FIG. 4. Stress-strain curves for the system in Fig. 1, deformed at different temperatures. The strain rate $(\dot{\varepsilon})$ is $5 \times 10^{8} \mathrm{~s}^{-1}$ except at $0 \mathrm{~K}$, where no strain rate can be defined (see Sec. IV D). We see a clear softening at increasing temperatures.

The use of CNA makes dislocations, grain boundaries and stacking faults visible in the simulation. Intrinsic stacking faults appear as two adjacent 111 planes of hcp atoms, extrinsic stacking faults are two 111 planes of hcp atoms separated by a single 111 plane of fcc atoms, whereas twin boundaries will be seen as a single 111 plane of hep atoms. Dislocation cores and grain boundaries consist of atoms in the "other" class, although grain boundaries also contain a low number of hcp atoms.

When analyzing simulations made at finite temperatures, the lattice vibrations may interfere with the CNA and it is necessary to precede the CNA analysis by a short minimization.

\section{RESULTS}

During the deformation, we calculate the average stress in the system as a function of the strain. Figure 4 shows the stress-strain curves for the same system at different temperatures. We clearly see a softening with increasing temperature. Rather large fluctuations are seen in the curves. These are mainly thermal fluctuations and fluctuations due to single major "events" in the systems (e.g., the nucleation of a dislocation). These fluctuations are only visible due to the small system size.

Figures 5 and 6 show the obtained stress-strain curves from simulations at 0 and $300 \mathrm{~K}$, respectively. We see a linear elastic region followed by a plastic region with almost constant stress. Similar results are found for palladium at $0 \mathrm{~K}$ (Fig. 7). Each stress-strain curve shown in the figures is obtained by averaging over a number of simulations with different (randomly produced) grain structures with the same average grain size. A set of stress-strain curves from individual simulations are shown in Fig. 8.

One of the rationales using a minimization procedure (i.e., a zero-temperature simulation) to study the deformations was the hope that the system would evolve through a series of local energy minima, separated by discrete events when the applied deformation causes the minima to disappear. In this way, the simulation would have resulted in a unique deformation history for any given sample. However, the deformation turned out to happen through a very large number of 


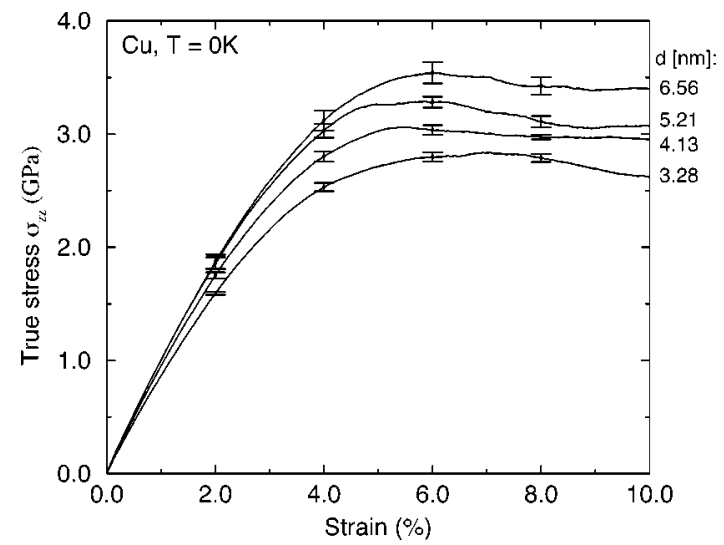

FIG. 5. Stress-strain curves for nanocrystalline copper at $0 \mathrm{~K}$. Each curve is the average over seven simulations with a given average grain size, the error bars indicate the uncertainty of the average $(1 \sigma)$. Adapted from Ref. 21.

very small processes, that could not be individually resolved by this procedure. One symptom of this is that the individual curves in Fig. 8 are not completely reproducible. Any even minor change in the minimization procedure, or a perturbation of the atomic coordinates, will result in a slightly different path through configuration space, and in different fluctuations in the stress-strain curves. Those differences are suppressed when average stress-strain curves are calculated, as in Fig. 5, and would also disappear as the system size (and thus the number of grains) increase.

\section{A. Young's modulus}

Young's modulus $(E)$ is the slope of the stress-strain curve in the linear region. Young's modulus is found to be around $90-120 \mathrm{GPa}$ at $0 \mathrm{~K}$ and it is increasing with increasing grain size.

When calculating Young's modulus from the simulation data, a compromise must be made between getting enough

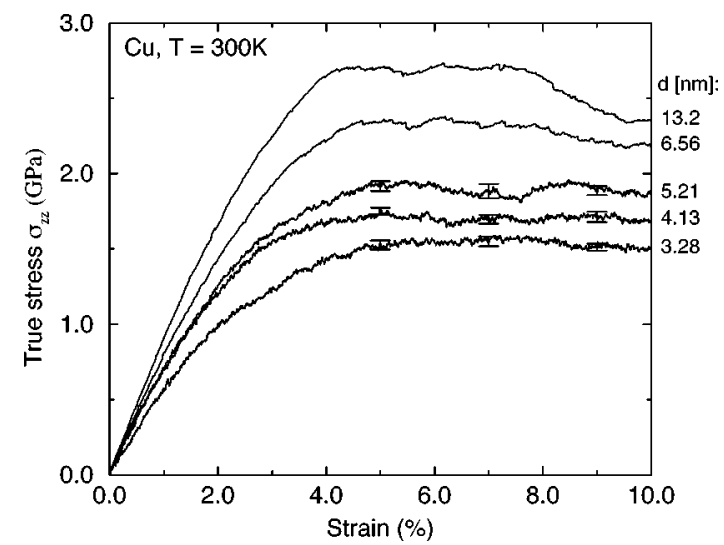

FIG. 6. Stress-strain curves for nanocrystalline copper at $300 \mathrm{~K}$ for varying grain size. The curves for grain sizes $d \leqslant 5.21 \mathrm{~nm}$ are the average of four simulations with a system size of 100000 atoms and a strain rate of $5 \times 10^{8} \mathrm{~s}^{-1}$. The simulations with $d \geqslant 6.56 \mathrm{~nm}$ were made with a system size of 1000000 atoms and a strain rate of $10^{9} \mathrm{~s}^{-1}$. The influence of this change in the strain rate is minimal, see Sec. IV D and Fig. 16. One simulation was performed with $d=6.56 \mathrm{~nm}$, two with $d=13.2 \mathrm{~nm}$. The thermal fluctuations are less pronounced in the simulations with the larger system size.

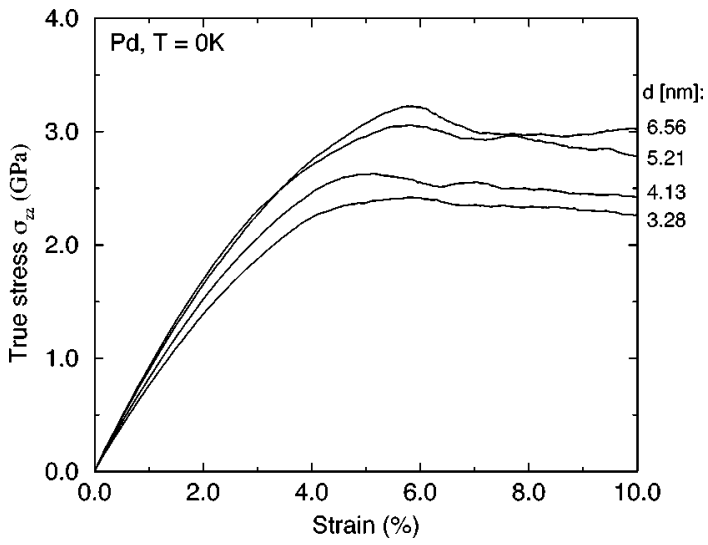

FIG. 7. Stress-strain curves for nanocrystalline palladium at $0 \mathrm{~K}$. Each curve is the average over two simulations with a given average grain size.

data point for a reliable fit, and staying within the clearly linear region. We have found that a reasonable compromise is to use data in the interval $\varepsilon<0.3 \%$, this ensures that we have enough data for a reliable fit, but results in a slight underestimate of the Young's modulus, as some plastic deformation is beginning in this interval. A smaller fitting interval could be used for the zero temperature simulations, but for consistency we used the same interval for all simulations.

Figure 9 shows Young's modulus for a single system with grain size $d=5.2 \mathrm{~nm}$, simulated at different temperatures. The observed temperature dependence of $E$ is approximately $-60 \pm 18 \mathrm{MPa} / \mathrm{K}$, which is somewhat larger than what has been observed experimentally $(-40 \mathrm{MPa} / \mathrm{K})$ in copper with a grain size of $200 \mathrm{~nm} .^{56}$ This may be because the Young's modulus of the grain boundaries is more temperature sensitive than in the bulk, or it may be due to increased "creep" (thermally activated grain boundary sliding) in the highertemperature simulations; see the discussion in Sec. IV D.

\section{B. Yield and flow stress}

The onset of plastic deformation is usually described by the yield stress $\sigma_{y}$, traditionally defined as the stress where the strain is 0.002 larger than what would be expected from extrapolation from the elastic region. In these simulations the

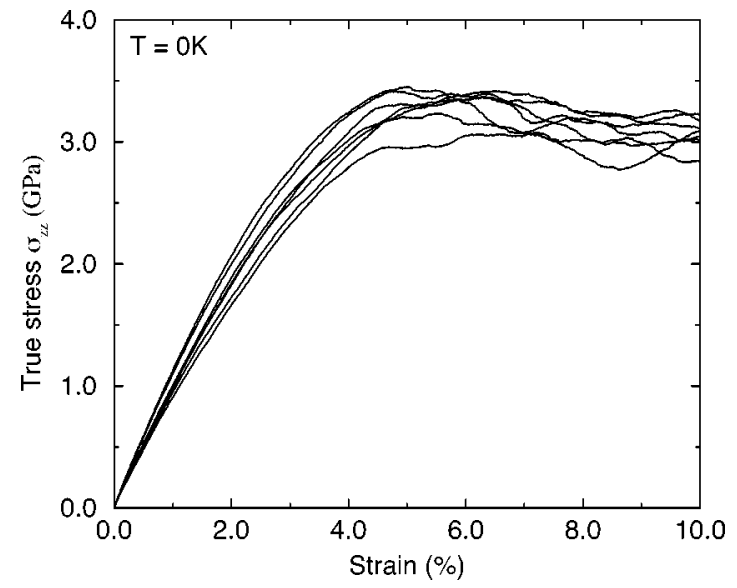

FIG. 8. Individual stress-strain curves for seven simulations of nanocrystalline copper with an average grain size of $5.21 \mathrm{~nm}$. 


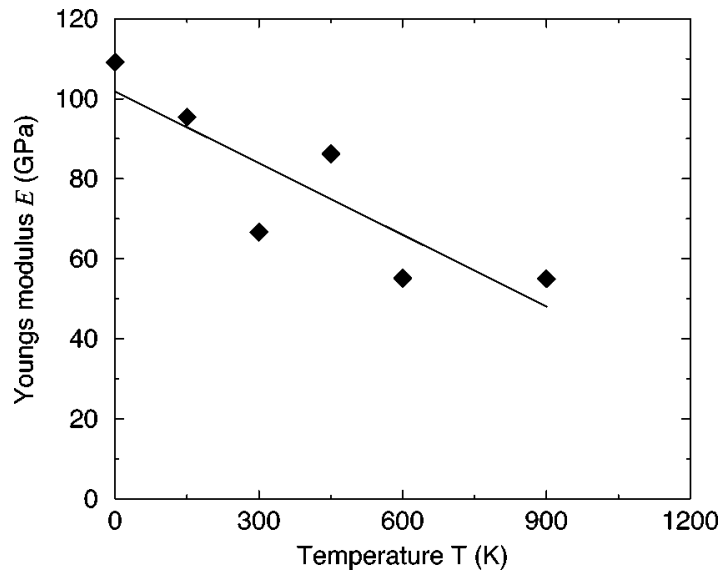

FIG. 9. Young's modulus as a function of temperature for a sample with an average grain size of $5.2 \mathrm{~nm}$. The Young's modulus at $1200 \mathrm{~K}$ is close to zero, but could not be determined due to very large fluctuations; see Fig. 4.

stress continues to increase after the yield point has been reached, until it reaches a plateau (the flow stress) and becomes constant or slightly decreasing.

The difficulties leading to an underestimate of the Young's modulus thus leads to a slight overestimate of the yield stress, and also leads to rather large uncertainties on the yield stress. The flow stress is a far more well-defined quantity. The variation of the yield and flow stresses with temperature is seen in Fig. 10.

Figure 11 shows the dependence of the yield and flow stress on the grain size at $0 \mathrm{~K}$ and at $300 \mathrm{~K}$. A clear reverse Hall-Petch effect is observed, i.e., a softening of the material as the grain size is reduced, as discussed in a previous paper. $^{21}$

\section{Structural changes}

Figure 1 shows the structural changes during $10 \%$ deformation. Some stacking faults have appeared in the grains, they are caused by partial dislocations (Shockley partials) nucleating at the grain boundaries and moving through the grains.

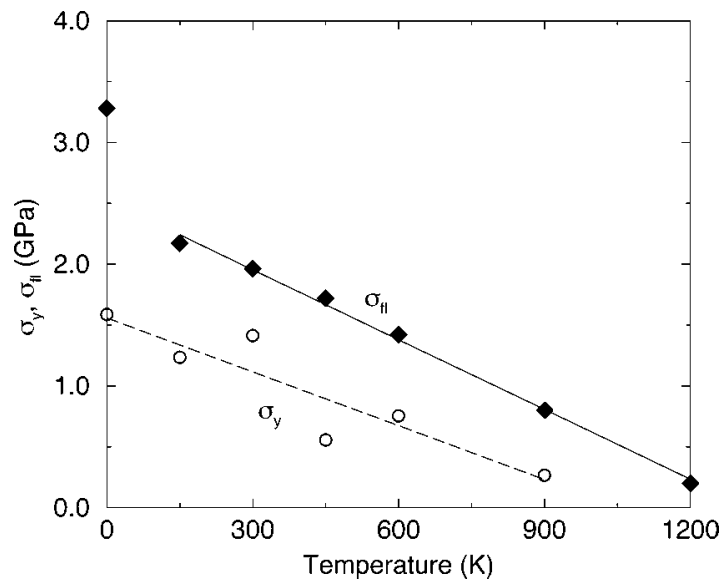

FIG. 10. Yield and flow stresses as a function of temperature. No yield stress is given for $T=1200 \mathrm{~K}$, as fluctuations are too large for a meaningful determination.

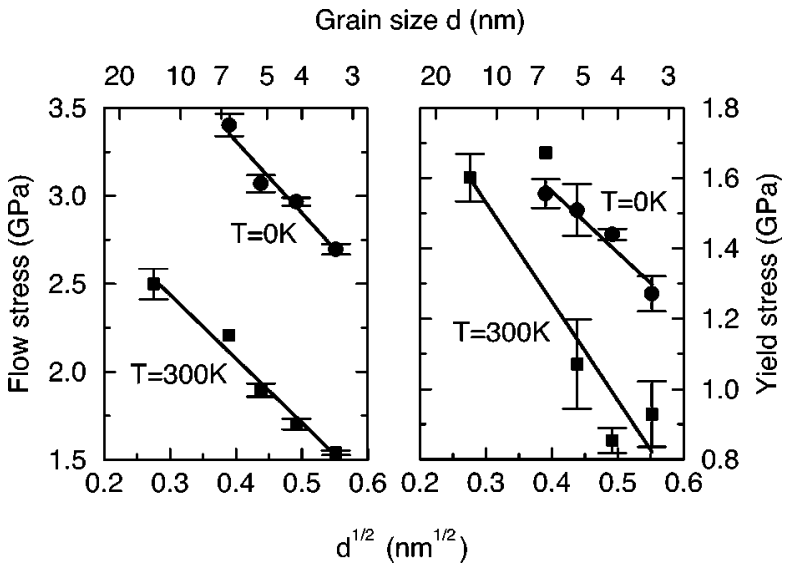

FIG. 11. Hall-Petch plot of the simulations at $0 \mathrm{~K}$ and at $300 \mathrm{~K}$, showing a reverse Hall-Petch relationship between the grain size and both the yield- and flow stress. Each point is the average of four values, except for the simulations at $300 \mathrm{~K}$ with grain sizes of 6.56 and $13.2 \mathrm{~nm}$, where only one and two simulations were performed. The error bars indicate the $1 \sigma$ statistical uncertainty.

The radial distribution function (Fig. 2) has been changed somewhat by the deformation. The peaks have been broadened; this is mainly caused by the anisotropic stress fields in the sample. The "background level" between the first two peaks has increased a little, indicating a larger amount of disorder in the system. Increased disorder is also seen in Fig. 1(b), where the grain boundaries appear to have become slightly thicker compared to the initial configuration. This is confirmed by Fig. 12, showing the number of atoms in different local configurations before and after the deformation. We see how more atoms are neither fcc nor hcp after the deformation than before.

The grain boundaries do not appear to increase as much in thickness at $300 \mathrm{~K}$ as they do at $0 \mathrm{~K}$. Figure 13 shows the change in the fraction of atoms in different local environ-

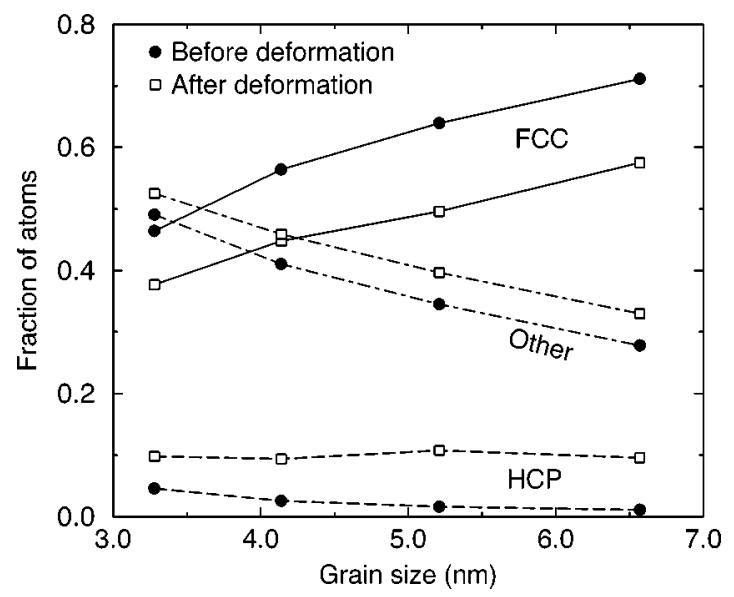

FIG. 12. The fraction of atoms in a given local crystal structure as a function of the grain size. Two curves are given for each type of atoms, one for the initial configurations (filled circles) and another showing how the fractions have changed after 10\% deformation (open squares). We see how the fraction of atoms in the grain boundaries (marked "Other') decreases with increasing grain size, and how more atoms are in grain boundaries and stacking faults (hcp) after the deformation than before. 


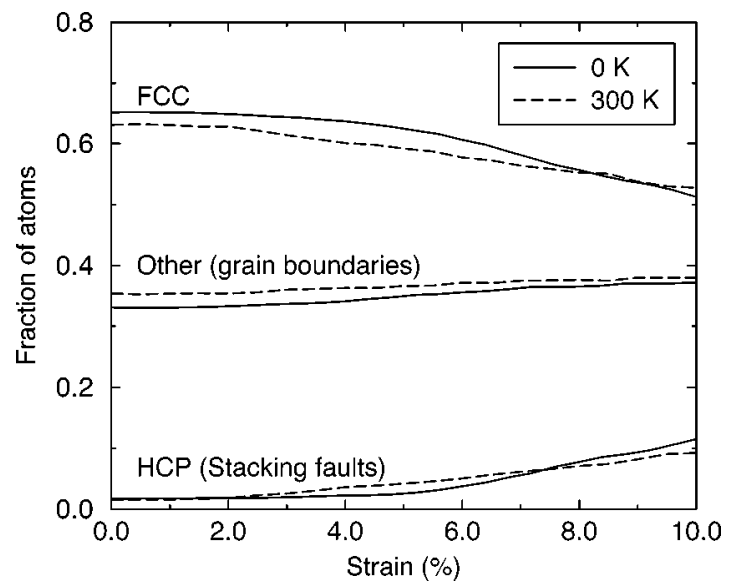

FIG. 13. Development of the number of atoms in different local environments as a function of the strain, for a single simulation at $0 \mathrm{~K}$ and at $300 \mathrm{~K}$.

ments during the deformation of the same system at $0 \mathrm{~K}$ and at $300 \mathrm{~K}$. In both cases we clearly see an increase in the number of hcp atoms (stacking faults) due to the motion of dislocations through the grains. We also see an increase of the number of atoms in the grain boundaries, in particular at $0 \mathrm{~K}$. The increase appears to be caused by the deformation in the grain boundaries. Apparently, the local disorder introduced in this way is partially annealed out at $300 \mathrm{~K}$.

The number of atoms near stacking faults (atoms in hcp symmetry) is also seen to increase strongly during the deformation (Figs. 1, 12, and 13). The stacking faults appear as partial dislocations move through the system, and they are thus the signature of dislocation activity. At zero temperature, we do not observe cases where a second partial dislocation erases the stacking faults (we observe only a very few atoms changing from local hcp order to local fcc order). We can therefore use the total number of hcp-ordered atoms to estimate an upper bound on the amount of plastic deformation caused by the dislocations.

If a dislocation with Burgers vector $\vec{b}$ runs through the entire system, the dimensions of the system are changed by $\vec{b}$ and the strain $\varepsilon_{z z}$ is thus $b_{z} L_{z}^{-1}$, where $b_{z}$ is the $z$ component of the Burgers vector and $L_{z}$ is the dimension of the system in the $z$ direction. If the dislocation only passes through a part of the system, the resulting deformation is reduced by $A \cos \phi\left(L_{x} L_{y}\right)^{-1}$, where $A$ is the area of the slip, $L_{x}$ and $L_{y}$ are the lateral dimensions of the system, and $\phi$ is the angle between the slip plane and the $x y$ plane. The contribution from a slip plane to the $z z$ component of the strain is thus $\varepsilon_{z z}=\left(b_{z} L_{z}^{-1}\right) A \cos \phi\left(L_{x} L_{y}\right)^{-1}$.

The maximal value of $b_{z}$ is $b \sin \phi$, where $b=|\vec{b}|$, since $\vec{b}$ lies in the slip plane. The maximal strain from the slip thus becomes $\varepsilon_{\max }=b A(2 V)^{-1}=a A(2 \sqrt{6} V)^{-1}$ for $\phi=\pi / 4$, as the Burgers vector of a Shockley partial is $b=a / \sqrt{6}$, where $a$ is the lattice constant and $V$ is the volume of the simulation cell. A slip plane of area $A$ results in two $\{111\}$ planes of hcp atoms, i.e., $4 A\left(\sqrt{3} a^{2}\right)^{-1}$ atoms. The total system contains $4 \mathrm{Va}^{-3}$ atoms, so the fraction of hcp atoms becomes $n$ $=A a(\sqrt{6} V)^{-1}$. Hence a fraction $n$ of hep atoms can at most have resulted in a strain of $\varepsilon_{\max }=2^{-3 / 2} n$. As the simulation generate at most $9 \%$ hcp atoms during $10 \%$ deformation, we get that $\varepsilon_{\max } \approx 3 \%$, provided that all slip planes and Burgers vectors are ideally aligned. It is therefore clear that the main deformation mode is not by dislocation motion.

Figures 14 and 15 illustrate how the main part of deformation has taken place. The atoms are colored according to their motion relative to the global stretching of the system. We clearly see that the upper parts of the grains have moved down and the lower parts up, relative to what would be expected in a homogeneous deformation. This shows that the grains do not stretch as much as in a homogeneous deformation. On the other hand, it is seen that significant deformation has happened in the grain boundaries, as the atoms typically are moving up on one side and down on the other side of a grain boundary. An analysis of that deformation shows that it is in the form of a large number of apparently uncorrelated small slipping events, where a few atoms (or a few tens of atoms) move relatively to reach other ${ }^{21}$ i.e., not in the form of collective motion in the grain boundaries. A minor part of the plastic deformation is in the form of dislocation motion inside the grains. The slip planes are clearly seen in Fig. 14, in particular in the large grain in the upper left part of the figure, where two dislocations have moved through the grain, and a third is on its way near one of the previous slip planes. The main deformation mode appears to be the same at zero and at finite temperatures.

\section{Strain rate}

As the time scale of molecular dynamics is set by the atomic motion, only very brief periods of time can be simulated. For the size of systems discussed here $1 \mathrm{~ns}$ (200000 time steps) is for all practical purposes an upper limit, although for repeated simulations $0.1 \mathrm{~ns}$ is a more realistic limit. One consequence of the short time scale is that very high strain rates are required to get any reasonable deformation within the available time

The finite-temperature simulations presented in this paper were performed at a strain rate of $\dot{\varepsilon}=5 \times 10^{8} \mathrm{~s}^{-1}$, unless otherwise is stated. This is very high, but still the ends of the system separates at velocities far below the speed of sound.

In order to investigate the influence of the strain rate, we simulated the same deformation of the same system using different strain rates in the range $2.5 \times 10^{7} \mathrm{~s}^{-1}-1.0 \times 10^{10}$ $\mathrm{s}^{-1}$; see Fig. 16. A strong dependence on the strain rate is seen for strain rates above $1 \times 10^{9} \mathrm{~s}^{-1}$. Below this "critical strain rate" the strain rate dependence on the stress-strain curves is far less pronounced. Figure 17 confirms this impression. It shows the yield and flow stress as a function of the strain rate. Experiments on ultrafine-grained $(d \approx 300$ $\mathrm{nm}) \mathrm{Cu}$ and $\mathrm{Ni}$ show a clear strain rate dependence on the yield and flow stresses at high strain rates. ${ }^{57}$

Perhaps surprisingly, the Young's modulus appears to depend on the strain rate as well (Fig. 16). This indicates that some kind of plastic deformation occurs in the "linear elastic" region. This is confirmed by stopping a simulation while the system still appears to be in the elastic region, and then allowing it to contract until the stresses are zero. The system does not regain the original length: plastic deformation has occurred.

To examine the time scale over which this deformation occurs, a configuration was extracted from the simulation at 

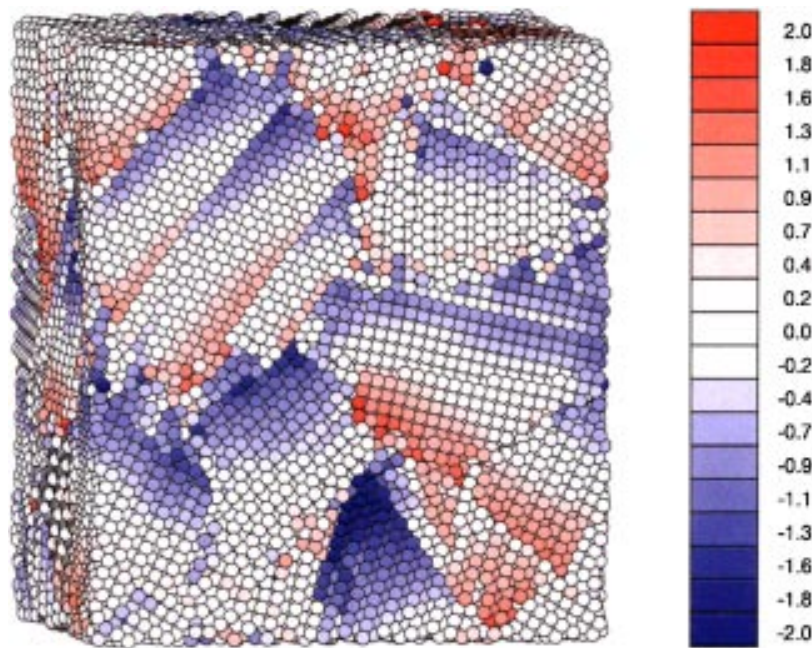

FIG. 14. (Color) The same system as in Fig. 1(b), but with the atoms colors according to their motion relative to the homogeneous deformation. Blue atoms have moved downwards relative to what would be expected if the deformation had been homogeneous and elastic; red atoms have moved upwards. The scale indicates how far the atoms have moved (in angstroms) during the $10 \%$ deformation. The grains are seen to be blue in the upper part, and red in the lower part, indicating that the grains have not been strained as much as the system. This indicates that a major part of the deformation has been in the grain boundaries. Several dislocations have moved through the large grain in the upper-left corner. Their slip planes are clearly seen
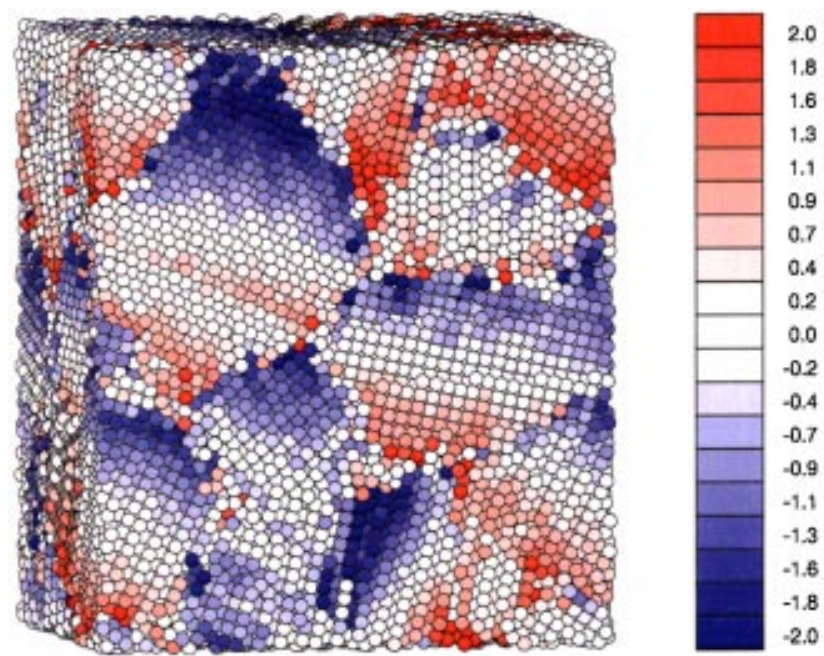

FIG. 15. (Color) The same system as in Fig. 14, but after deformation at $300 \mathrm{~K}$; see the caption of that figure for a description of the color coding. The main deformation is seen to be in the grain boundaries, as was the case at $0 \mathrm{~K}$.

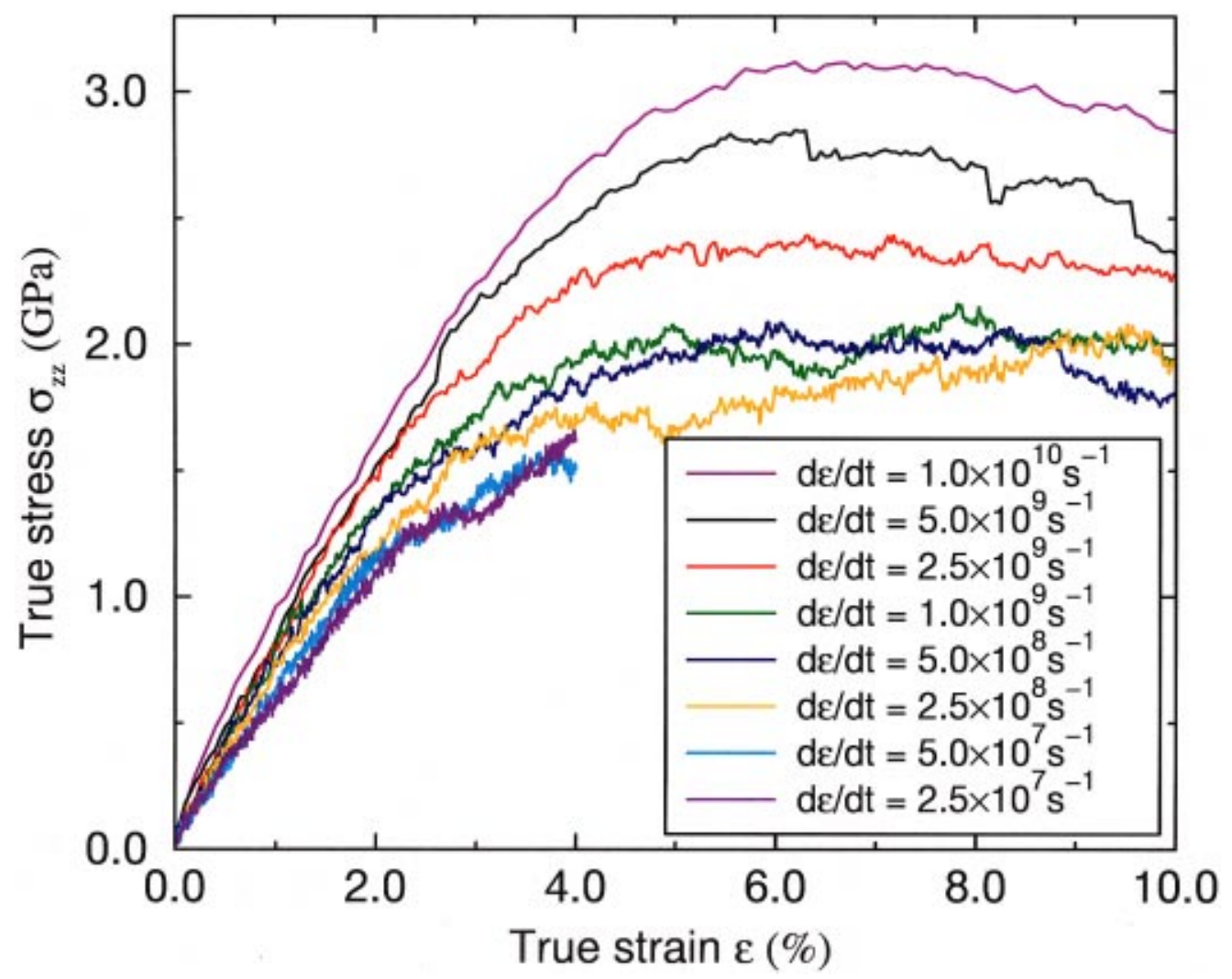

FIG. 16. (Color) The effect of varying the strain rate. Stress-strain curves are shown for simulations of the same system at the same temperature $(300 \mathrm{~K})$, but with varying strain rates. A strong influence of strain rate is seen for strain rates above $\dot{\varepsilon}=10^{9} \mathrm{~s}^{-1}$; below that value the strain rate dependence is less pronounced. The simulations at the two lowest strain rates were stopped after $4 \%$ deformation. 


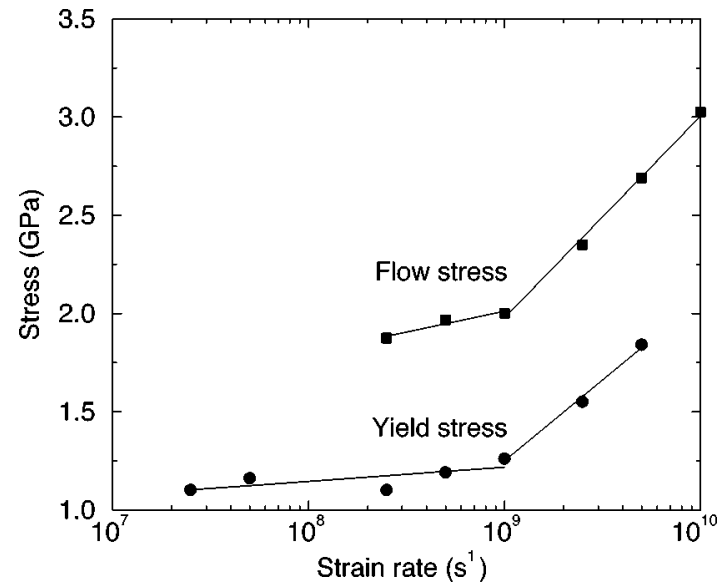

FIG. 17. Summary of the effect of varying the strain rate. Both the yield stress and the flow stress are seen to vary with the strain rate $(\dot{\varepsilon})$, strongest for $\dot{\varepsilon}>10^{9} \mathrm{~s}^{-1}$.

$\dot{\varepsilon}=2.5 \times 10^{7} \mathrm{~s}^{-1}$ after $0.4 \%$ deformation. The system was held at a fixed length for $300 \mathrm{ps}$ while the stress was monitored; see Fig. 18. The stress is seen to decrease with a characteristic time of approximately 100 ps. By plotting the atomic motion in a plot similar to Figs. 14 and 15, it is seen that the relaxation is due to small amounts of plastic deformation in the grain boundaries. The consequence of this is that the systems do not have time to relax completely during the simulations, explaining the observed strain rate dependence. In order to allow for complete relaxation of the systems, strain rates far below what is practically possible with MD simulations are required.

As another consequence of the short time-scale, slower processes will not be seen in the simulations. In particular, most diffusional processes will be unobservable (this would also be the case in experiments performed at these high strain rates). However, measurements of diffusional creep (Coble creep) in nanocrystalline metals indicate that diffusional creep is not a large effect. ${ }^{58,59}$

The $T=0 \mathrm{~K}$ simulations were performed using a minimization procedure. In such simulations time is not defined,

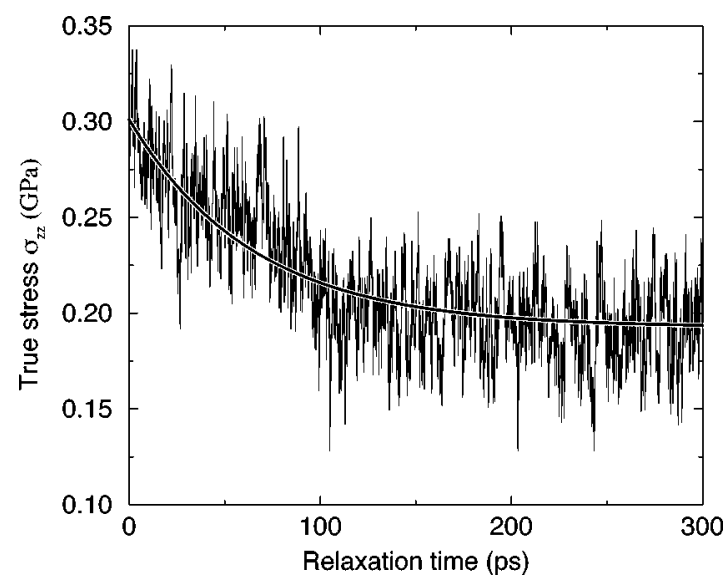

FIG. 18. Sample relaxation in the "elastic" region. The deformation is stopped after $0.4 \%$ deformation at $\dot{\varepsilon}=2.5 \times 10^{7} \mathrm{~s}^{-1}$ and $T=300 \mathrm{~K}$. The stress is seen to decrease with a characteristic time of $\sim 100 \mathrm{ps,} \mathrm{stabilizing} \mathrm{on} \mathrm{a} \mathrm{value} \mathrm{of} 2 / 3$ the original stress. The thick black line is an exponential fit.

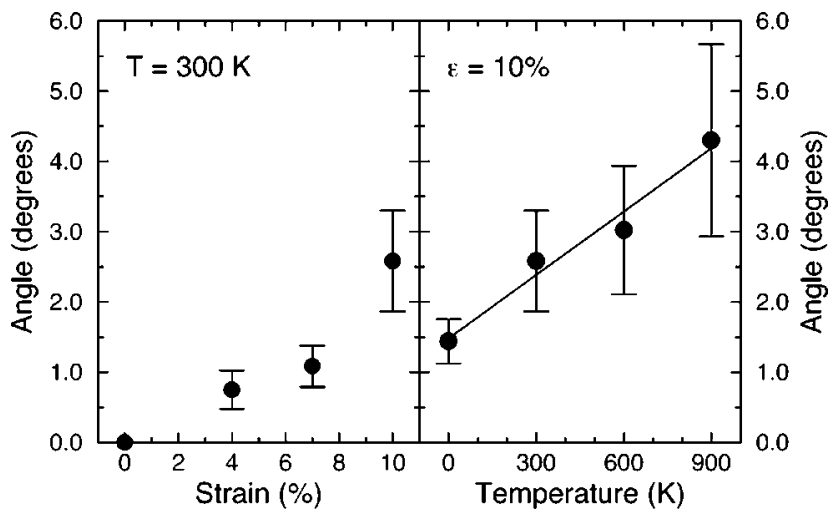

FIG. 19. Grain rotation as a function of strain and temperature. The rotation of five randomly selected grains were followed in simulations at different temperatures. The average grain size is 5.2 $\mathrm{nm}$. The error bars indicate the $1 \sigma$ spread in the rotations.

since we are not solving an equation of motion. In a similar way, time should not be relevant in an experiment performed truly at zero temperature, since there will be no thermally activated processes, and thus no way the sample can leave a local energy minimum. So the strain rate will not have an effect on the results, providing it is low enough to prevent a heating of the sample, and providing the minimization procedure is fully converged. The minimization simulations can thus be seen as a model for idealized experiments at zero temperature in the low strain rate limit, where there is time for the heat generated by the deformation to be removed.

\section{E. Grain rotation}

Grain rotation has previously been reported in simulations of nanocrystalline nickel. ${ }^{17} \mathrm{We}$ have investigated the rotation of the grains during some of the simulations, the results are summarized in Fig. 19. The figure shows the rotation of five randomly selected grains as a function of strain and temperature. The rotations were identified by a three-dimensional Fourier transform of the positions of the atoms in the grains.

We see that the grain rotation increases with increasing temperature. There is a large variation between how much the individual grains rotate. The grains with the largest rotations keep the same axis of rotation during the entire deformation, whereas the grains that only rotate a little have a varying axis of rotation. Probably some grains are in a local environment where a significant rotation results in an advantageous deformation of the sample which reduces the stress. Other grains are randomly rotated as the many small deformation processes in the grain boundaries occur.

\section{F. Porosity}

As the observed reverse Hall-Petch effect is often explained as an artifact of sample porosity (see Sec. V B), we found it relevant to study how pores influence the mechanical properties. The void structure in experimentally produced samples is usually not well known, so we chose to study several different types of voids. In all cases the voids resulted in a reduction of both the Young's modulus and the flow stress; see Fig. 20. 

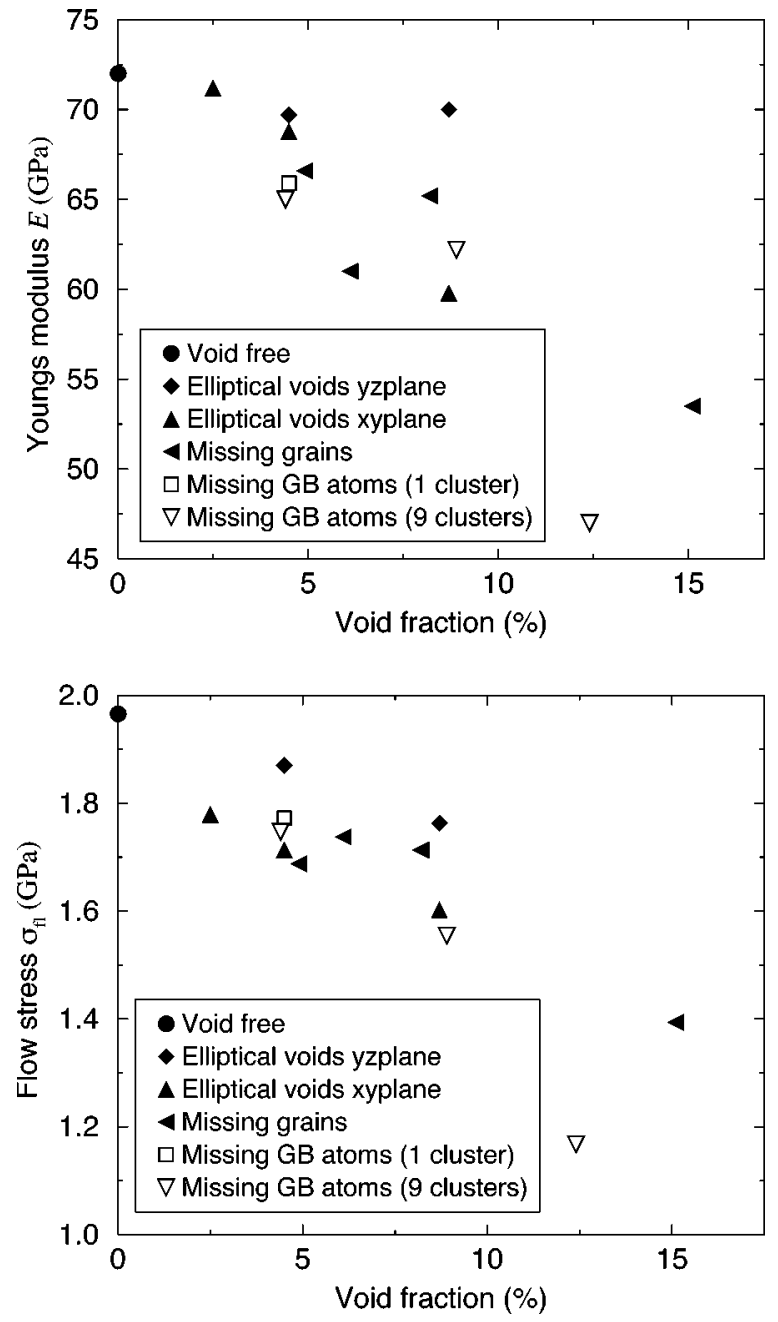

FIG. 20. Effect of voids on the Young's modulus (top) and on the flow stress (bottom), showing a decrease of both quantities with increasing void fraction. The voids were generated by removing selected atoms, the "void fraction" is the fraction of atoms removed. Different methods were used to select the atoms to be removed; see Sec. IV F.

Elliptical voids. These cracklike voids were created by removing all atoms within an oblate ellipsoid with an axis ratio of 3.16. The short axis can be oriented along the pulling direction (the crack is in the $x y$ plane) or perpendicular to it (the crack is in the $y z$ axis). The former orientation corresponds to cracks that are activated by the applied stress field, the effect of these cracks is therefore expected to be much larger than the effect of the "inactive" cracks. This is clearly seen in Fig. 20.

Missing grains. There have been reports of pore sizes comparable to (and proportional to) the grain size. ${ }^{60,61}$ To mimic this, we have tried to remove whole grains from the system. As the grains are approximately equiaxed, it is not surprising that the effect of removing a grain is intermediate between the effects of removing ellipsoids in the two orientations, provided that approximately the same number of atoms are removed.

Missing grain-boundary atoms. In samples experimentally produced by compacting a powder, it is reasonable to assume that the porosity will mainly be in the form of (possibly gas-filled) voids between the grains. There is also some experimental evidence that this is indeed the case. ${ }^{61,62}$ To mimic this, we have removed all atoms in the grain boundaries within one or nine spherical regions in the sample, creating one large or nine small voids in the grain boundaries. This type of voids have the largest effect on the materials properties, giving a reduction of $35-40 \%$ in the Young's modulus and flow stress for a $12.5 \%$ porosity. It seems rather natural that a large effect is obtained with the voids concentrated in the grain boundaries since we know that the main part of the deformation is carried by these boundaries.

\section{DISCUSSION}

\section{A. Elastic properties}

From our simulations we get values of the Young's modulus around $100 \mathrm{GPa}$ at $0 \mathrm{~K}$. This should be compared to the value found for single crystals using this potential: $150 \mathrm{GPa}$ at $0 \mathrm{~K}$ (Hill average calculated from the anisotropic elastic constants $C_{11}=173 \mathrm{GPa}, C_{12}=116 \mathrm{GPa}, C_{44}=91$ $\mathrm{GPa}$ ). The experimental value for macrocrystalline copper is $124 \mathrm{GPa}$ at $300 \mathrm{~K} \cdot{ }^{63}$ A significant reduction of the Young's modulus is thus seen in the nanocrystalline phase.

A similar reduction of Young's modulus is seen in simulations of nanocrystalline metals grown from a molten phase. ${ }^{11}$ The low value is usually explained by a large volume fraction of the atoms being in the grain boundaries. ${ }^{64}$ These atoms experience a different atomic environment, which could result in a reduction of the elastic moduli similar to what is seen in amorphous metals. This local reduction of the elastic constants in grain boundaries is confirmed by atomistic simulations. ${ }^{65}$

Experimental measurements of the Young's modulus of high quality (i.e., low-porosity) samples of nanocrystalline copper and palladium show a reduction in Young's modulus of at most a few percent when correcting for the remaining porosity. ${ }^{60}$ These results were obtained for significantly larger grain sizes than were used in the simulations. The reduction of Young's modulus that we observe in these simulations will be difficult to detect experimentally, due to the much lower volume fraction of atoms in the grain boundaries for typical grain sizes in high quality samples $(\geq 20$ $\mathrm{nm})$.

\section{B. Reverse Hall-Petch effect}

A reverse Hall-Petch effect in nanocrystalline copper was first observed in nanocrystalline $\mathrm{Cu}$ and $\mathrm{Pd}$ by Chokshi et al. in $1989 .{ }^{31}$ Since then, there have been numerous observations of softening at very small grain sizes. ${ }^{32-34}$

The reverse Hall-Petch effect seems to depend strongly on the sample preparation technique used and on the sample history, perhaps indicating that in most cases the reverse Hall-Petch effect is caused by various kinds of defects in the samples. Surface defects alone have been shown to be able to decrease the strength of nanocrystalline metals by a factor of $5,{ }^{58,66}$ and recent studies have shown that even very small amounts of porosity can have a dramatic effect on the strength. ${ }^{60,67}$ Improved inert gas condensation techniques ${ }^{68}$ have reduced the porosity resulting in samples with densities above $98 \%$ of the fully dense value. In these samples the ordinary Hall-Petch effect is seen to continue down to grain 
sizes around $15 \mathrm{~nm} .{ }^{60}$ There are only few data points below that grain size, but apparently no further increase in the hardness is seen. It is suggested that most of the observations of a reverse Hall-Petch effect in nanocrystalline metals are a result of poor sample quality. ${ }^{60}$ This impression is supported by literature studies ${ }^{32,69}$ indicating that the reverse Hall-Petch effect is mainly seen when the grain size is varied by repeated annealing of a single sample, whereas an ordinary Hall-Petch relationship is seen when as-prepared samples are used.

However, there does seem to be a deviation from the Hall-Petch effect for grain sizes below approximately $15 \mathrm{~nm}$, where the Hall-Petch slope is seen to decrease or vanish in samples produced with various techniques. This is seen in $\mathrm{Cu}$ samples produced by inert gas condensation followed by warm compaction (sample densities above 98\%) (Ref. 60) and in electroplated $\mathrm{Ni}$ (claimed to be porosity free). ${ }^{70,71}$

There are theoretical arguments for expecting that the Hall-Petch relation ceases to be valid for grain sizes below $\sim 20 \mathrm{~nm}$ : as the grain size becomes too small, dislocation pileups are no longer possible, and the usual explanation for Hall-Petch behavior does not apply. ${ }^{59,72,73}$

Many models have been proposed to explain why a reverse Hall-Petch effect is sometimes seen. Chokshi et al. ${ }^{31}$ proposed that enhanced Coble creep, i.e., creep by vacancy diffusion in the grain boundaries, should result in a softening at the smallest grain sizes as the creep rate increases with decreasing grain size $(d)$ as $d^{-3}$. Direct measurements of the creep rate have however ruled this out. ${ }^{59,74}$

It has been suggested that the grain boundaries in nanocrystalline metals have a different structure, making them more transparent to dislocations than "ordinary" grain boundaries. ${ }^{75-77}$ If it becomes possible for the dislocations to run through several grains as the grain size is reduced, the Hall-Petch relations would break down. In our simulations, we have not observed dislocations moving through more than one grain.

If the Hall-Petch effect is explained by appealing to dislocation sources in the grain boundaries, the Hall-Petch relationship is expected to break down when the grain sizes becomes so low that there are no longer dislocation sources in all grain boundaries ${ }^{78}$ (assuming a constant density of dislocation sources in the grain boundaries).

Hahn and co-workers ${ }^{79,80}$ suggest that the reverse HallPetch effect is caused by deformation in the grain boundaries. If a grain boundary slides, stress concentrations build up where the grain boundary ends, limiting further sliding. Substantial sliding on a macroscopic scale occurs when sliding occurs on slide planes consisting of many aligned grain boundaries; the sliding is hindered by the roughness of the slide plane due to its consisting of many grain boundaries. As the grain size is reduced and becomes comparable to the grain-boundary width, the roughness of such slide planes decreases and the stress required for mesoscopic sliding decreases. This would result in a reverse Hall-Petch effect. They estimate the transition from normal to reverse HallPetch effect to occur at grain sizes near $50 \mathrm{~nm}$ for $\mathrm{Cu}^{79}$

The simulations reported in the present paper indicate that the main deformation mechanism at these grain sizes is in- deed sliding in the grain boundaries. However, it is not clear if the proposed "collective" sliding events are occurring, it appears that sliding occurs on individual grain boundaries, and that the resulting stress buildup is relieved through dislocation motion in the grains. There is a competition between the ordinary deformation mode (dislocations) and the grainboundary sliding. As the grain size is increased, the dislocation motion is eventually expected to dominate, and we expect a transition to a behavior more like what is seen in coarse-grained materials, including a normal Hall-Petch effect. The transition is beyond what can currently be simulated at the atomic scale, but we do see a weak increase in the dislocation activity when the grain size is increased: The increase in the fraction of hcp atoms during a simulation is increasing slightly with the grain size (Fig. 12).

\section{CONCLUSIONS}

Molecular dynamics and related techniques have been shown to be a useful approach to study the behavior of nanocrystalline metals. We have in detail investigated the plastic deformation of nanocrystalline copper, and shown that the main deformation mode is sliding in the grain boundaries. The sliding happens through a large number of small, apparently uncorrelated events, where a few grain boundary atoms (or a few tens of atoms) move past each other. It remains the main deformation mechanism at all grain sizes studied (up to $13 \mathrm{~nm}$ ), even at zero temperature. As the grain boundaries are the main carriers of the deformation, decreasing the number of grain boundaries by increasing the grain size leads to a hardening of the material; a reverse Hall-Petch effect. This is observed in the simulations, both for $T=0 \mathrm{~K}$ and for $T$ $=300 \mathrm{~K}$.

The Young's moduli of the nanocrystalline systems are found to be reduced somewhat compared to the experimental value for polycrystalline copper with macroscopic grain sizes, decreasing with decreasing grain size. This indicates that the grain boundaries are elastically softer than the grain interiors. The Young's modulus is decreasing with increasing temperature at a rate somewhat above what is seen experimentally in coarser-grained copper.

Pores in the samples have a large effect on both the Young's modulus and the flow stress. This effect is enhanced if the pores are mainly in the grain boundaries, as one could expect in samples produced experimentally by inert gas condensation. Sample porosity can explain a large number of experiments showing reverse Hall-Petch effect, but the softening due to grain boundary sliding may be important for high-quality samples with grain sizes close to the lower limit of what can be reached experimentally.

\section{ACKNOWLEDGMENTS}

Major parts of this work were financed by The Danish Technical Research Council (STVF) through Grant No. 9601119. Parallel computer time was financed by the Danish Research Councils through Grant No. 9501775. The Center for Atomic-scale Materials Physics is sponsored by the Danish National Research Council. 
*Also at: Materials Research Department, Ris $\varnothing$ National Laboratory, DK-4000 Roskilde, Denmark.

${ }^{\dagger}$ Present address: INFM and SISSA, Via Beirut 2-4, I-34014 Grignano (TS), Italy.

${ }^{1}$ F. F. Abraham, Europhys. Lett. 38, 103 (1997).

${ }^{2}$ V. Bulatov, F. F. Abraham, L. Kubin, B. Devincre, and S. Yip, Nature (London) 391, 669 (1998).

${ }^{3}$ S. J. Zhou, D. L. Preston, P. S. Lomdahl, and D. M. Beazley, Science 279, 1525 (1998).

${ }^{4}$ T. Rasmussen, K. W. Jacobsen, T. Leffers, and O. B. Pedersen, Phys. Rev. B 56, 2977 (1997).

${ }^{5}$ T. Rasmussen, K. W. Jacobsen, T. Letters, O. B. Pedersen, S. G. Srinivasan, and H. Jónsson, Phys. Rev. Lett. 79, 3676 (1997).

6 R. W. Siegel and G. E. Fougere, in Nanophase Materials: Synthesis-Properties-Applications, Vol. 260 of NATO-ASI Series E: Applied Sciences, edited by G. C. Hadjipanayis and R. W. Siegel (Kluwer, Dordrecht, 1994), pp. 233-261.

${ }^{7}$ R. W. Siegel, Mater. Sci. Forum 235-238, 851 (1997).

${ }^{8}$ D. G. Morris and M. A. Morris, Mater. Sci. Forum 235-238, 861 (1997).

${ }^{9}$ D. G. Morris, Mechanical Behavior of Nanostructured Materials, Vol. 2 of Materials Science Foundations (Trans Tech Publications, Ütikon-Zürich, 1998).

${ }^{10}$ D. Chen, Comput. Mater. Sci. 3, 327 (1995).

${ }^{11}$ S. R. Phillpot, D. Wolf, and H. Gleiter, J. Appl. Phys. 78, 847 (1995).

${ }^{12}$ S. R. Phillpot, D. Wolf, and H. Gleiter, Scr. Metall. Mater. 33, 1245 (1995).

${ }^{13}$ H. Zhu and R. S. Averback, Mater. Manuf. Processes 11, 905 (1996).

${ }^{14}$ P. Keblinski, R. Phillpot, D. Wolf, and H. Gleiter, Acta Mater. 45, 987 (1997).

15 G. D'Agostino and H. Van Swygenhoven, in Metastable Phases and Microstructures, edited by R. Borman, G. Mazzone, R. D. Shull, R. S. Averback, and R. F. Ziolo, MRS Symposia Proceedings No. 400 (Materials Research Society, Pittsburgh, 1996), p. 293.

${ }^{16}$ H. Van Swygenhoven and A. Caro, Appl. Phys. Lett. 71, 1652 (1997).

${ }^{17}$ H. Van Swygenhoven and A. Caro, Nanostruct. Mater. 9, 669 (1997).

${ }^{18}$ H. Van Swygenhoven and A. Caro, Phys. Rev. B 58, 11246 (1998).

${ }^{19}$ H. Van Swygenhoven, M. Spaczér, and A. Caro, Nanostruct. Mater. 10, 819 (1998).

${ }^{20}$ H. Van Swygenhoven, M. Spaczér, and A. Caro, in Microscopic Simulation of Interfacial Phenomena in Solids and Liquids, edited by S. R. Phillpot, P. D. Bristowe, D. G. Stroud, and J. R. Smith, MRS Symposia Proceedings No. 492 (Materials Research Society, Pittsburgh, 1998), pp. 357-362.

${ }^{21}$ J. Schiøtz, F. D. Di Tolla, and K. W. Jacobsen, Nature (London) 391, 561 (1998).

22 J. Schiøtz, T. Vegge, F. D. Di Tolla, and K. W. Jacobsen, in Modelling of Structure and Mechanics of Materials from Microscale to Product, Proceedings of the 19th Ris $\phi$ International Symposium on Materials Science, edited by J. V. Carstensen et al. (Risø National Laboratory, Roskilde, 1998), pp. 133-148.

23 J. Schiøtz, T. Vegge, and K. W. Jacobsen, in Multiscale Modeling of Materials, edited by V. Bulatov, T. D. de la Rubia, R. Phillips, E. Kaxiras, and N. Ghoniem, MRS Symposia Proceed- ings No. 538 (Materials Research Society, Pittsburgh, 1999), p. 299.

${ }^{24}$ J. R. Weertman, D. Farkas, K. Hemker, H. Kung, M. Mayo, R. Mitra, and H. Van Swygenhoven, MRS Bull. 24, 44 (1999).

${ }^{25}$ E. O. Hall, Proc. Phys. Soc. London, Sect. B 64, 747 (1951).

${ }^{26}$ N. J. Petch, J. Iron Steel Inst., London 174, 25 (1953).

${ }^{27}$ J. C. M. Li and Y. T. Chou, Metall. Trans. A 1, 1145 (1970).

${ }^{28}$ Z. Jiang, J. Lian, and B. Baudelet, Acta Metall. Mater. 43, 3349 (1995).

${ }^{29}$ L. H. Friedman and D. C. Chrzan, Phys. Rev. Lett. 81, 2715 (1998).

${ }^{30}$ L. H. Friedman and D. C. Chrzan, Philos. Mag. A 77, 1185 (1998).

${ }^{31}$ A. H. Chokshi, A. Rosen, J. Karch, and H. Gleiter, Scr. Metall. 23, 1679 (1989).

${ }^{32}$ G. E. Fougere, J. R. Weertman, R. W. Siegel, and S. Kim, Scr. Metall. Mater. 26, 1879 (1992).

${ }^{33}$ T. R. Malow, C. C. Koch, P. Q. Miraglia, and K. L. Murty, Mater. Sci. Eng., A 252, 36 (1998).

${ }^{34}$ T. Yamasaki, P. Schloßmacher, K. Ehrlich, and Y. Ogino, Nanostruct. Mater. 10, 375 (1998).

${ }^{35}$ R. W. Siegel, J. Phys. Chem. Solids 55, 1097 (1994).

${ }^{36}$ H. Natter, M. Schmelzer, and R. Hempelmann, J. Mater. Res. 13, 1186 (1998).

37 A. Okabe, B. Boots, and K. Sugihara, Spatial Tesselations: Concepts and Applications of Voronoi Diagrams (Wiley, Chichester, 1992).

${ }^{38}$ S. Kumar, S. K. Kurtz, J. R. Banavar, and M. G. Sharma, J. Stat. Phys. 67, 523 (1992).

${ }^{39}$ O. H. Nielsen, J. P. Sethna, P. Stoltze, K. W. Jacobsen, and J. K. Nørskov, Europhys. Lett. 26, 51 (1994).

40 J. Schiøtz, Ph.D. thesis, Technical University of Denmark, Lyngby, Denmark, 1995.

${ }^{41}$ E. A. Stern, R. W. Siegel, M. Newville, P. G. Sanders, and D. Haskel, Phys. Rev. Lett. 75, 3874 (1995).

${ }^{42}$ K. W. Jacobsen, J. K. Nørskov, and M. J. Puska, Phys. Rev. B 35, 7423 (1987).

${ }^{43}$ K. W. Jacobsen, P. Stoltze, and J. K. Nørskov, Surf. Sci. 366, 394 (1996).

44 M. P. Allen and D. J. Tildesley, Computer Simulation of Liquids (Claredon Press, Oxford, 1987).

${ }^{45}$ W. C. Swope, H. C. Andersen, P. H. Berens, and K. R. Wilson, J. Chem. Phys. 76, 637 (1982).

46 In order to implement periodic boundary conditions all atomic coordinates are internally represented in a "scaled space," where coordinates are between 0 and 1 . The real coordinates are obtained by multiplying with a matrix $M$. The system is deformed by changing the relevant components of $M$, not by changing the scaled-space coordinates.

47 The minimization method is described under the name Molecular Dynamics Minimization in P. Stoltze, Simulation Methods in Atomic Scale Materials Physics (Polyteknisk Forlag, Lyngby, Denmark, 1997).

${ }^{48}$ T. Egami, K. Maede, and V. Vitek, Philos. Mag. A 41, 883 (1980).

${ }^{49}$ J. R. Ray and A. Rahman, J. Chem. Phys. 80, 4423 (1984).

${ }^{50}$ J. R. Ray and A. Rahman, J. Chem. Phys. 82, 4243 (1985).

${ }^{51}$ D. H. Tsai, J. Chem. Phys. 70, 1375 (1979).

${ }^{52}$ J. F. Lutsko, J. Appl. Phys. 64, 1152 (1988).

${ }^{53}$ K. S. Cheung and S. Yip, J. Appl. Phys. 70, 5688 (1991).

${ }^{54}$ H. Jónsson and H. C. Andersen, Phys. Rev. Lett. 60, 2295 (1988). 
${ }^{55}$ A. S. Clarke and H. Jónsson, Phys. Rev. E 47, 3975 (1993).

${ }^{56}$ A. B. Lebedev et al., Mater. Sci. Eng., A 203, 165 (1995).

${ }^{57}$ G. T. Gray III et al., Nanostruct. Mater. 9, 477 (1997).

${ }^{58}$ G. W. Nieman, J. R. Weertman, and R. W. Siegel, J. Mater. Res. 6, 1012 (1991).

${ }^{59}$ T. G. Nieh and J. Wadsworth, Scr. Metall. Mater. 25, 955 (1991).

${ }^{60}$ P. G. Sanders, C. J. Youngdahl, and J. R. Weertman, Mater. Sci. Eng., A 234-236, 77 (1997).

${ }^{61}$ P. G. Sanders, J. A. Eastman, and J. R. Weertman, Acta Mater. 46, 4195 (1998).

62 S. R. Agnew et al., in Modelling of Structure and Mechanics of Materials from Microscale to Product, Proceedings of the 19th Ris $\phi$ International Symposium on Materials Science, edited by J. V. Carstensen et al. (Risø National Laboratory, Roskilde, 1998), pp. 1-14.

${ }^{63}$ K. A. Gschneidner, Solid State Phys. 16, 275 (1964).

${ }^{64}$ T. D. Shen, C. C. Koch, T. Y. Tsui, and G. M. Pharr, J. Mater. Res. 10, 2892 (1995).

${ }^{65}$ M. D. Kluge, D. Wolf, J. F. Lutsko, and S. R. Phillpot, J. Appl. Phys. 67, 2370 (1990).

${ }^{66}$ J. R. Weertman, Mater. Sci. Eng., A 166, 161 (1993).

${ }^{67}$ P. G. Sanders, J. A. Eastman, and J. R. Weertman, Acta Mater. 45, 4019 (1997).

${ }^{68}$ P. G. Sanders, G. E. Fougere, L. J. Thompson, J. A. Eastman, and J. R. Weertman, Nanostruct. Mater. 8, 243 (1997).

69 J. Grilhe, in Mechanical Properties and Deformation Behavior of
Materials Having Ultra-Fine Microstructures, Vol. 233 of NATO-ASI Series E: Applied Sciences, edited by M. Nastasi, D. M. Parker, and H. Gleiter (Kluwer, Dordrecht, 1993), pp. 255-286.

${ }^{70}$ A. M. El-Sherik, U. Erb, G. Palumbo, and K. T. Aust, Scr. Metall. Mater. 27, 1185 (1992).

${ }^{71}$ N. Wang, Z. Wang, K. T. Aust, and U. Erb, Mater. Sci. Eng., A 237, 150 (1997).

${ }^{72}$ C. S. Pande, R. A. Masumura, and R. W. Armstrong, Nanostruct. Mater. 2, 323 (1993).

${ }^{73}$ N. Wang, Z. Wang, K. T. Aust, and U. Erb, Acta Metall. Mater. 43, 519 (1995).

${ }^{74}$ G. W. Nieman, J. R. Weertman, and R. W. Siegel, Scr. Metall. Mater. 24, 145 (1990).

${ }^{75}$ R. Z. Valiev, F. Chmelik, F. Bordeaux, G. Kapelski, and B. Baudelet, Scr. Metall. Mater. 27, 855 (1992).

${ }^{76}$ J. Lian, B. Baudelet, and A. A. Nazarov, Mater. Sci. Eng., A 172, 23 (1993).

${ }^{77}$ K. Lu and M. L. Sui, Scr. Metall. Mater. 28, 1465 (1993).

78 S. Li, L. Sun, and Z. Wang, in Strength of Materials, edited by H. Oikawa, K. Maruyama, S. Takeuchi, and M. Yamaguchi (The Japan Institute of Metals, 1994), pp. 873-876.

${ }^{79}$ H. Hahn and K. A. Padmanabhan, Philos. Mag. B 76, 559 (1997).

${ }^{80}$ H. Hahn, P. Mondal, and K. A. Padmanabhan, Nanostruct. Mater. 9, 603 (1997). 